\title{
Neural Network Model Predictive Control (NNMPC) Design for UPFC
}

\author{
S.A. Al-MAWSAWI, A. HAIDER, Q. ALFARIS \\ Department of Electrical and Electronics Engineering \\ University of Bahrain, College of Engineering \\ KINGDOM OF BAHRAIN \\ aalmossawi@uob.edu.bh, aakbar@uob.edu.bh, qalfares@hotmail.com
}

\begin{abstract}
Neural Network Model Predictive Control (NNMPC) is like almost like the model predictive control but the used inboard plant is designed based on using the concept of the artificial neural network to predict the behavior of the plant. The predicted values are fed to the optimizer in order to obtain better control variables. This type of controller will be used instead of the conventional controller in the most versatile FACTS devices, which is the Unified Power Flow Controller (UPFC). UPFC has the capability of controlling the transmission line parameters and consequently the flow of the active and reactive power in the transmission line. So, this type of adaptive controller, which is based on Artificial Neural Network (ANN) concept, will be implemented in UPFC, and will be investigated to ensure its robustness, effectiveness and the capability to accommodate any sudden load change in the system of Single Machine to Infinite Bus (SMIB). In addition, the dynamic performance of NNMPC will be compared with another type of adaptive controller scheme called Model Productive Controller (MPC).
\end{abstract}

Key-Words: FACTS, UPFC, NNMPC, MPC, ANN, SMIB

Received: July 14, 2020. Revised: September 21, 2020. Accepted: November 2, 2020. Published: November 16, 2020.

\section{Introduction}

Unified Power Flow Controller (UPFC) has the ability to control, independently or simultaneously, all parameters that affect the active and reactive power flow on the transmission line such as the voltage magnitude, impedance and phase angle [1]. The UPFC have a positive impact in enhancing the power quality and system performance [2] and [3]. An important issue in the design of controllers for such a device is robustness, i.e., the controller should achieve the desired damping over a wide range of system operating conditions [4]. The controllers which are being used in UPFC are very important to control all those parameters as desired. An adaptive scheme called Neural Network Model Predictive Control will be used in this study. NNMPC is on Artificial Neural Network (ANN) concept. ANN is considered as a model of how the human brain works. A biological neural network is an essential part of human brain. It is a highly complex network with the ability to process huge amounts of information simultaneously. The input impulses travel via the sensory portion of the peripheral nervous system to the central nervous system for higher level interpretation to response and convey the action through the peripheral nervous system to relevant part in the human body. So, human brain contains of an enormous number of nerve cells and neurons. The combinations of these cells are together creating a very complex network of signal transmission. Each cell collects inputs from all other neural cells it is connected to and if the collected cell information reaches a certain threshold, then it will be conveyed to all the cells it is connected to. So, the interconnection of the large number of neurons in the Biological neurons network architecture will allow a rapid communication spanning throughout all areas of the body. Although, Biological neural networks are complex, but Artificial Neural Network model will be basic structure representation as shown in Table 1 [5] 
TABLE 1

Basic Structure of Biological Neuron

\begin{tabular}{|l|l|}
\hline Structure & Function \\
\hline Dendrites & Input \\
\hline Cell body & Integration \\
\hline Axon & Conduction \\
\hline $\begin{array}{l}\text { Pre-Synaptic } \\
\text { terminals }\end{array}$ & Output \\
\hline
\end{tabular}

ANNs, like human, learn by example. ANN is configured for a specific application, such as pattern recognition or data classification, through a learning process. Learning in biological systems involves adjustments to the synaptic connections that exist between the neurons. ANN can be trained after implementation and needs a trainer designed in hardware or software to provide punishments or rewards for the adopted weights. Each input has an associated weight ' $w$ ', which can be modified so as to model synaptic learning by using the concept of back propagation.

\section{Back Propagation Concept}

The ANN cell/unit computes some function ' $\mathrm{f}$ ' of the weighted sum of its inputs:

$y_{i}=f\left(\sum_{j} w_{i j} y_{j}\right)$

Its output, in turn, can serve as input to other units as illustrated in Fig. 1.

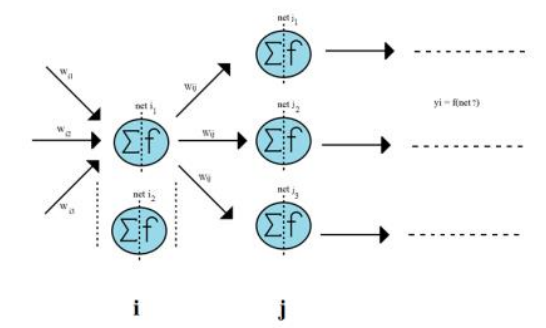

Fig.1. Artificial Neuron
The weighted sum $\sum_{\mathrm{j}} \mathrm{w}_{\mathrm{ij}} \mathrm{y}_{\mathrm{j}}$ is called the net input to the 'net'. Note that 'wij' refers to the weight from unit $j$ to unit ' $i$ '. The function ' $f$ ' is the unit's activation function as could be as linear, sigmoid, step, ...etc. So, in the feed forward neural network the inputs are multiplied by the weights then will be summed in the neural cell where the result of the summation will also pass through the activation function ' $\mathrm{f}$ '. The outcome from the neural cell will be multiplied again with the next weights and the process will continue up until the final result is obtained. One the final result is obtained it will be compared with the actual result in order to determine the error and train the model. Back propagation will be used to train the network. An example will be extracted from Fig 1, in order to clarify the concept and the equation that will be used in the feed forward and back propagation method. So, for simplicity one string which is in green colour will be analysed as illustrated in Fig. 2 .

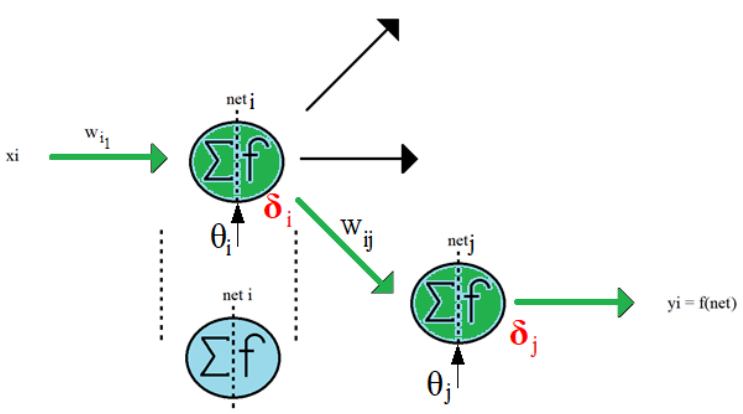

Fig.2. Data Flow in One String of Artificial Neuron

The calculation starts from the last output neuron all the way back to the input:

$$
\mathrm{Y}_{\mathrm{j}}=\mathrm{f}_{\mathrm{j}}\left(\mathrm{f}_{\mathrm{i}}\left(\mathrm{X}_{\mathrm{i}}^{*} \mathrm{~W}_{\mathrm{i} 1}\right) * \mathrm{~W}_{\mathrm{ij}}\right)
$$

Error $=\mathrm{Y}_{\text {desire }}-\mathrm{Y}_{\mathrm{j}}$

$$
\text { Gradient Error }\left(\delta_{\mathrm{j}}\right)=\frac{\partial \mathrm{Yj}}{\partial \mathrm{xi}} * \text { Error }
$$

The output from neuron ' $\mathrm{i}$ ' is

$$
\mathrm{Y}_{\mathrm{i}}=\mathrm{f}_{\mathrm{i}}\left(\mathrm{X}_{\mathrm{i}}^{*} \mathrm{~W}_{\mathrm{i} 1}\right)
$$

$$
\text { Gradient Error }\left(\delta_{\mathrm{i}}\right)=\frac{\partial \mathrm{Yi}}{\partial \mathrm{xi}} *\left(\mathrm{~W}_{\mathrm{ij}} * \delta_{\mathrm{j}}\right)
$$


After getting the gradient error 1 and 2 from equation number (4) and (6) respectively, the $\Delta \mathrm{W}$ and $\Delta \theta$ will be calculated in order to update the existing weights and biases.

$$
\begin{aligned}
& \Delta \mathrm{W}_{\mathrm{ij}}=\text { learning Rate }(\alpha) * \mathrm{Y}_{\mathrm{i}} * \delta_{\mathrm{j}} \\
& \Delta \mathrm{W}_{\mathrm{i} 1}=\text { learning Rate }(\alpha) * \mathrm{X}_{\mathrm{i}} * \delta_{\mathrm{i}} \\
& \Delta \theta_{\mathrm{j}}=\text { learning Rate }(\alpha) * \theta_{\mathrm{j}} * \delta_{\mathrm{j}} \\
& \Delta \theta_{\mathrm{i}}=\text { learning Rate }(\alpha) * \theta_{\mathrm{i}} * \delta_{\mathrm{i}}
\end{aligned}
$$

Hence the $\Delta \mathrm{W}$ and $\Delta \theta$ are obtained, the weights and biases will be updated as follows:

$$
\begin{aligned}
& \mathrm{W}_{\mathrm{ij}} \leftarrow \mathrm{W}_{\mathrm{ij}}+\Delta \mathrm{W}_{\mathrm{ij}} \\
& \mathrm{W}_{\mathrm{i} 1} \leftarrow \mathrm{W}_{\mathrm{i} 1}+\Delta \mathrm{W}_{\mathrm{i} 1} \\
& \theta_{\mathrm{j}} \leftarrow \theta_{\mathrm{j}}+\Delta \theta_{\mathrm{j}} \\
& \theta_{\mathrm{i}} \leftarrow \theta_{\mathrm{i}}+\Delta \theta_{\mathrm{i}}
\end{aligned}
$$

The next input will be introduced to the network and same procedure will be followed to obtain the outputs and correct the weights and biases.

\section{UPFC Study}

Gyupyi introduced the UPFC in 1991 [6]. It is composed of two voltage source converters linked by common d.c link as illustrated in Fig. 3.

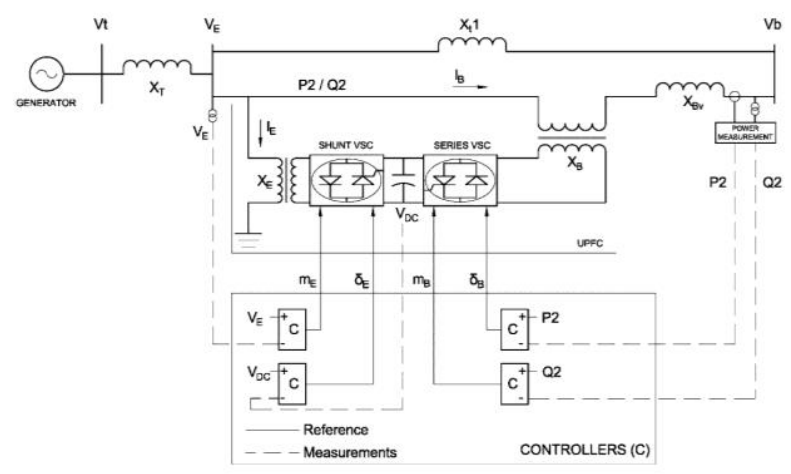

Fig.3. UPFC in SMIB

Mathematical models for the steady state and dynamic model will be needed in order to inspect the performance of the UPFC in the system. The steady state model is concerned to determine the initial condition of the system to perform the load flow analysis. While, the dynamic model will be performed to ensure that the performance of the UPFC and its controllers during disturbance and any sudden load changes are acceptable and met the expectations. A. Nabavi-Niaki and M. R. Iravani [7] the model is considered in this study. The system control variables are controlled via the amplitude of the modulation ratios $m_{E}$ and $m_{E}$ and the phase angle of the voltage source converter control signal $\delta_{E}$ and $\delta_{B}$. The control variables $m_{E}, m_{E}, \delta_{E}$ and $\delta_{B}$ are selected to be connected to the control output signal to control $V_{E}, Q_{2}, V_{d c}$ and $P_{2}$ respectively.

\section{System Study}

The UPFC is incorporated in a Single Machine to Infinite Bus (SMIB) system to test and analysis the entire system performance. Model number 1.0 of a synchronous generator with IEEE ST1A excitation system will be adopted as it is used in most of the dynamic studies of power system such as the studied performed by M. Abido [8], M. Abido et. Al. [9] and S. A. Alqallaf [10]. Matlab platform will be used to perform the system simulation.

\section{NNMPC Control Design}

\subsection{The Concept of NNMPC Controller}

Model Predicative Control (MPC) is widely used approach which relies on solving a numerical optimization problem online, but due to the complexity of nonlinear control problems it is in general necessary to apply various computational or approximative procedures for the solution. The main drawback of the MPC is that the optimization problem may computationally quite demanding for nonlinear systems. So, in order to reduce the on-line computational requirements, another approach is applied as off-line function approximators to represent the optimal control law such as artificial neural network [11]. Neural networks have been applied very successfully in the identification and control of dynamic systems. The universal approximation capabilities of the multilayer perception make it a popular choice for modelling of nonlinear systems and for implementing of nonlinear controllers. Two-layer networks, with sigmoid transfer functions in the hidden layer and linear transfer functions in the output layer, are universal approximators. The Neural Network Model Predictive Controller is based on the concept of the Artificial Neural Network. NNMPC uses a neural network model of a nonlinear plant to predict future plant performance. Then the controller calculates the control input which will optimize plant performance over a specified future time horizon. The training data were obtained from the nonlinear model of the system. The used model predictive control method was based on the receding horizon technique. The 
neural network model predicted the plant response over a specified time horizon. The predictions were used by a numerical optimization program to determine the control signal that minimizes performance criterion over the specified horizon [12]. The following steps will be followed to design the NNMPC:

1) System identification: the model predictive control is used to determine the neural network plant model. The prediction error between the plant output and the neural network output is used as the neural network training signal as shown in Fig. 4 [13-15].

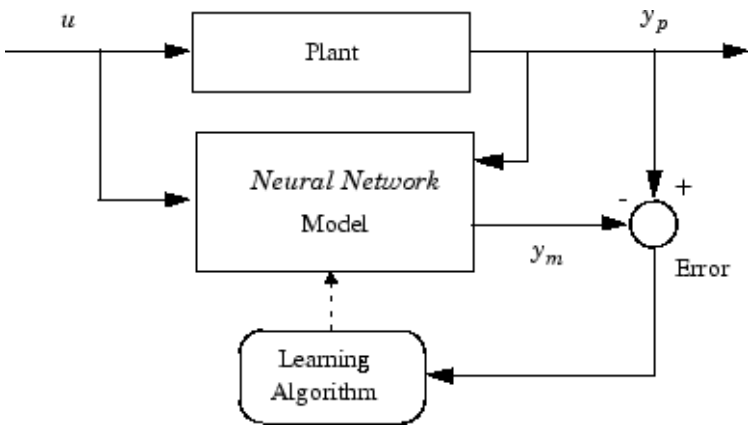

Fig.4. NNMPC System Identification

2) The neural network plant model to predict future performance: The past inputs and past plant outputs will be used to predict future values of the plant output as illustrate in Fig. 5. [12]. $u(t)$ is the system input, $y p(t)$ is the plant output, $y m(t)$ is the neural network model plant output, the blocks labeled TDL are tapped delay lines that store previous values of the input signal, IW $i, j$ is the weight matrix from the input $j$ to the layer $i . L W i, j$ is the weight matrix from the layer $\mathrm{j}$ to the layer $\mathrm{i}$.

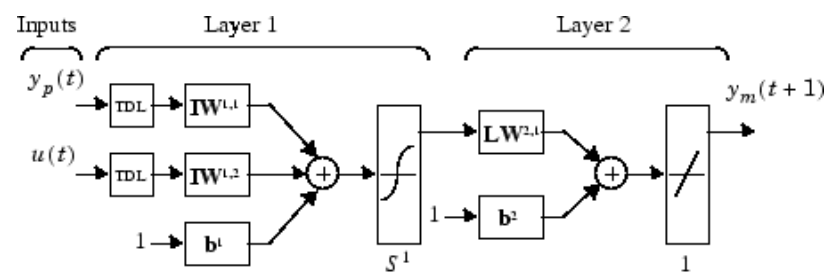

Fig.5. NNMPC prediction

3) Train the NNMPC by back propagation method.

4) Optimization algorithm will be used to determine the control signal that minimizes the cost function in equation (15) over the specified horizon.

$J=\sum_{j=N 1}^{N 2}(y r(t+j)-y m(t+j))^{2}+$

$\rho \sum_{j=1}^{N u}\left(u^{\prime}(t+j-1)-u^{\prime}(t+j-2)\right)^{2}$
Where, N1, N2, and Nu define the horizons over which the tracking error and the control increments are evaluated. $\mathrm{u}^{\prime}$ variable is the tentative control signal. $\mathrm{y}_{\mathrm{r}}$ is the desired response. $\mathrm{y}_{\mathrm{m}}$ is the network model response. $\rho$ value determines the contribution that the sum of the squares of the control increments has on the performance index.

5) The block diagram [16-20] in Fig. 6 shows the model predictive control process. The controller consists of the neural network plant model and the optimization block, where the optimization block determines the values of $\mathrm{u}^{\prime}$ that minimize $\mathrm{J}$ in equation (18), and then the optimal $u$ is input to the plant.

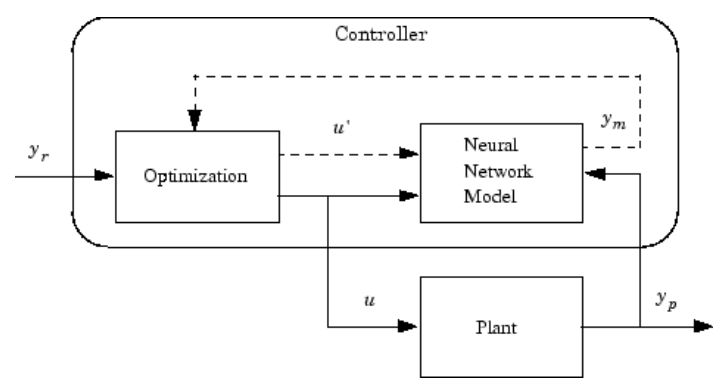

Fig.6. NNMPC Model Predictive Control Process

In this study the real power in line 2 is considered as a reference signal which will be fed to the NNMPC. The output of real power in line 2 from the SMIB will be also fed to the NNMPC in order to simulate and give the proper control signal to the plant as illustrated in Fig. 7.

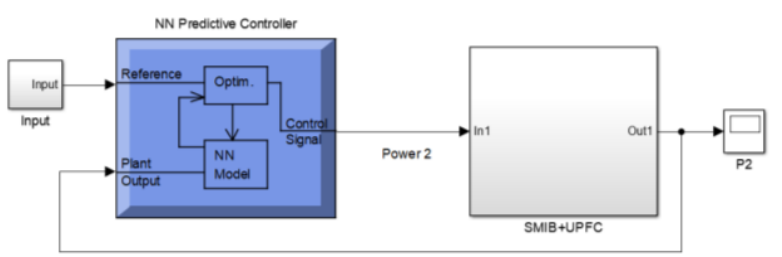

Fig.7. NNMPC Matlab Model

Part of NNMPC actions is to perform the system identification to determine the neural network plant model in order send the output to the optimization program to generate the control signal. In order to get an acceptable performance, the number of the neural network hidden layer was selected to be 30 and 10000 numbers of training samples were used to train the neural network model. 


\subsection{MPC Concept Design}

MPC refer to a type of computer control algorithms that utilize an explicit process model to predict the future response of the plant [7]. The concept behind MPC is that it takes the reference signals and the plant outputs and generate control outputs just like any other controller except it is using the inboard model of the plant to predict the behaviour of the plant in future by any of the following method such as Kalman Predictor, BJ model, ARX, ARMAX or ...etc.[8]. Future output predication is affected by the past state on future outputs, future inputs on future outputs and model mismatch.

The predicted behaviour of the plant will be fed to an optimizer to adjust of the value of the control outputs to make sure that the predicted plant outputs track the reference signals. MPC is considered as a popular controller in industrial applications because at every time step the process executed in the control algorithm, there is optimization involved to give better control outputs.

\subsection{Dynamic Response Performance of NNMPC}

\subsubsection{NNMPC Performance in Case of Sudden Step Change}

Figures 8 to 11 show the dynamic performance of NNMPC and MPC for the real power in line $2\left(\mathrm{P}_{2}\right)$, reactive power $\left(\mathrm{Q}_{2}\right)$, DC line voltage $\left(\mathrm{V}_{\mathrm{dc}}\right)$ and terminal line voltage $\left(\mathrm{V}_{\mathrm{Et}}\right)$ respectively. In this case, a sudden step change test $(-10 \%)$ at time second number 15 has been done for the real power $\left(\mathrm{P}_{2}\right)$. It can be seen that, both types of controllers are efficient to stabilize the system. Table 2 shows that, the dynamic performance of MPC is slightly better than NNMPC in raising time, setting time and overshoot for only in which parameter has been tested $\left(\mathrm{P}_{2}\right)$.

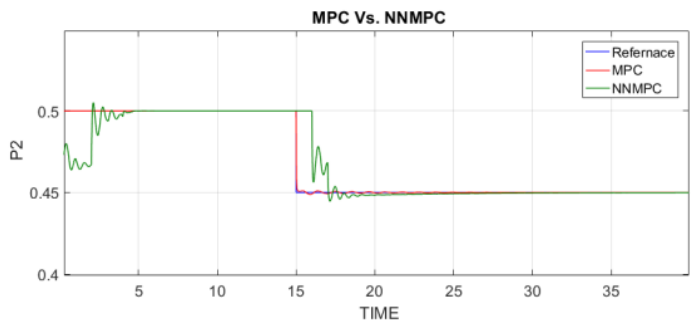

Fig.8. Real Power flow $\left(\mathrm{P}_{2}\right)$ in case of sudden step change $(-10 \%)$

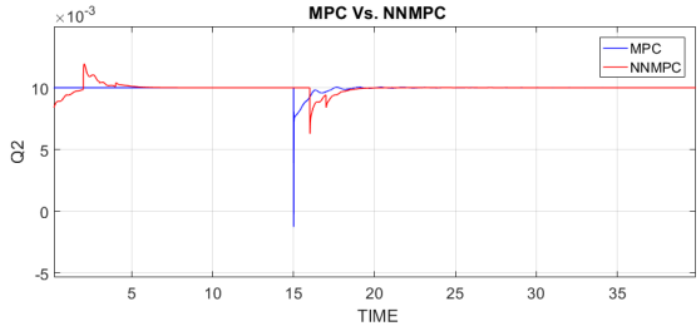

Fig.9. Reactive Power flow $\left(\mathrm{Q}_{2}\right)$ in case of sudden step change $(-10 \%)$

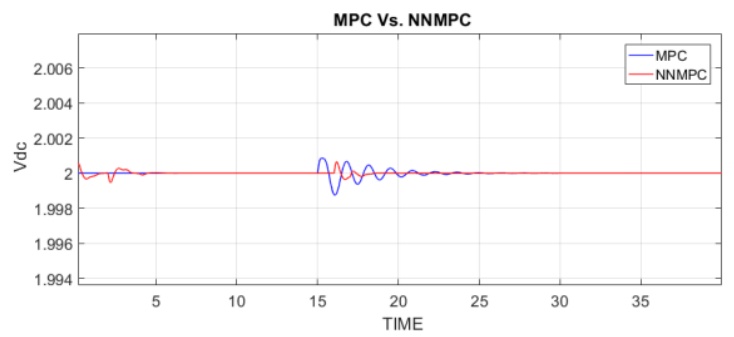

Fig.10. Dc Line Voltage $\left(\mathrm{V}_{\mathrm{dc}}\right)$ in case of sudden step change $(-10 \%)$

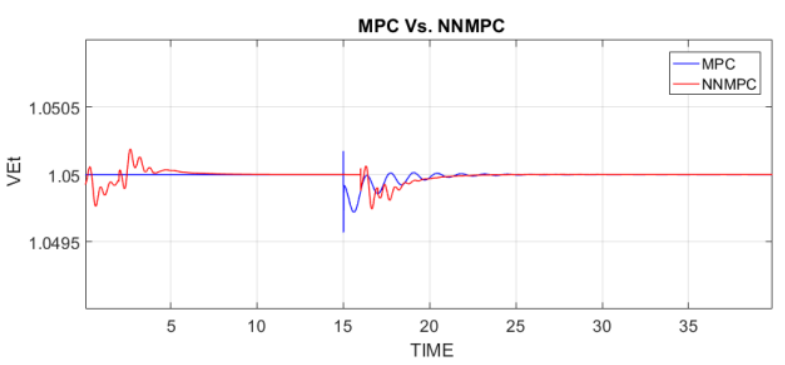

Fig.11. Terminal Voltage $\left(\mathrm{V}_{\mathrm{Et}}\right)$ in case of sudden step change $(-10 \%)$

Table. 2

Rating Score for each type of controller during ($10 \%)$ sudden step change for the real power $\mathrm{P}_{2}$

\begin{tabular}{|l|l|l|l|}
\hline & $\begin{array}{l}\text { Rise Time } \\
\text { (Sec.) }\end{array}$ & $\begin{array}{l}\text { Settling } \\
\text { Time (Sec.) } \\
(2 \%)\end{array}$ & $\begin{array}{l}\text { Overshoot } \\
(\%)\end{array}$ \\
\hline MPC & 0.09 & 1.4 & 0.18 \\
\hline $\begin{array}{l}\text { NNMP } \\
\text { C }\end{array}$ & 2.09 & 3.6 & 0.54 \\
\hline
\end{tabular}

In addition, it has been noticed from Fig. 12 that, the $10 \%$ reduction in power flow in line 2 is diverted to line number 1 in order to meet the total load required 
which is equal to 1 p.u. So, the power flow manoeuvre is achieved in this case satisfactorily.

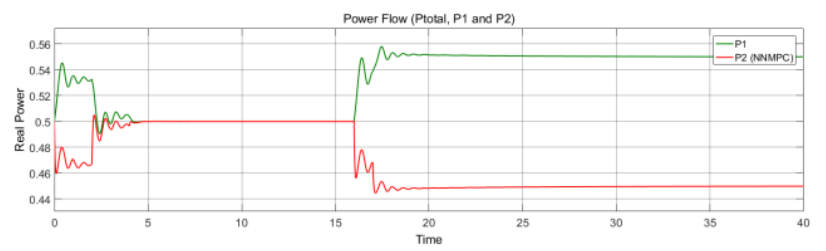

Fig.12. Real Power flow in line 1 and 2 by using NNMPC in case of sudden step change $(-10 \%)$

\subsubsection{NNMPC Performance in Case of Sudden System Disturbance}

Figures 13 to 16 show the dynamic performance of NNMPC and MPC for the real power in line $2\left(\mathrm{P}_{2}\right)$, reactive power $\left(\mathrm{Q}_{2}\right)$, DC line voltage $\left(\mathrm{V}_{\mathrm{dc}}\right)$ and terminal line voltage $\left(\mathrm{V}_{\mathrm{Et}}\right)$ respectively. In this case, a sudden system disturbance at time second number 70 has been done for the real power $\left(\mathrm{P}_{2}\right)$. It can be seen that, both types of controllers are responding to the system change satisfactorily. Table 3 shows that, the dynamic performance of MPC is much better than NNMPC in raising time, setting time and overshoot for only in which parameter has been tested $\left(\mathrm{P}_{2}\right)$.

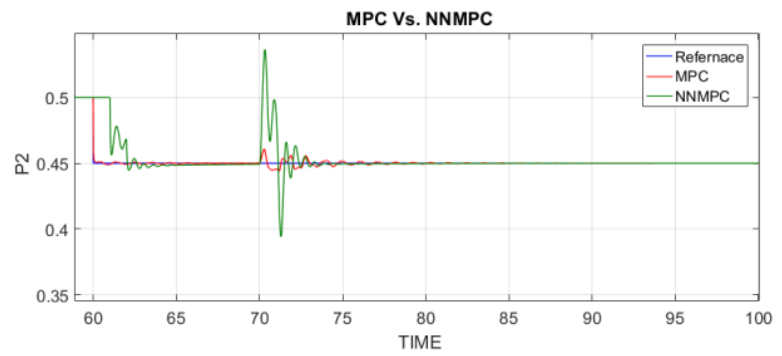

Fig.13. Real Power flow $\left(\mathrm{P}_{2}\right)$ in case of sudden system disturbance.

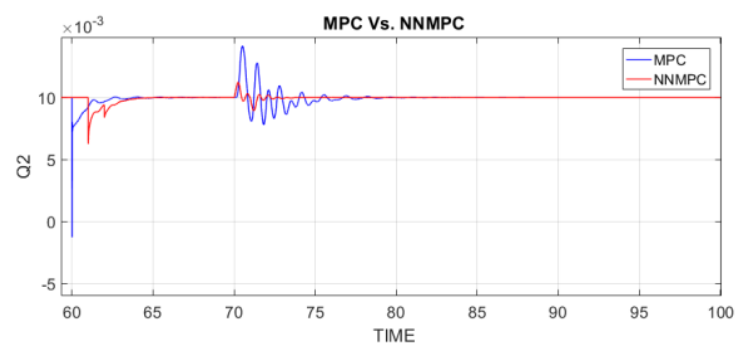

Fig.14. Reactive Power flow $\left(\mathrm{Q}_{2}\right)$ in case of sudden system disturbance

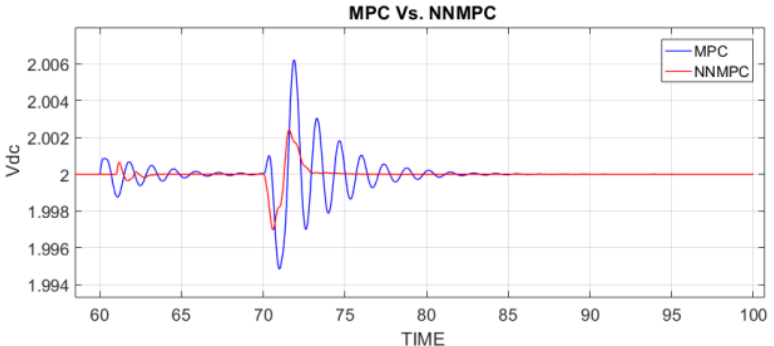

Fig.15. DC line voltage $\left(\mathrm{V}_{\mathrm{dc}}\right)$ in case of sudden system disturbance

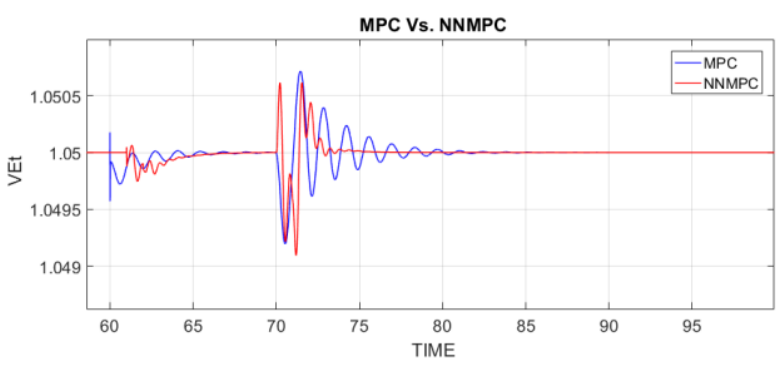

Fig. 16. Terminal voltage $\left(\mathrm{V}_{\mathrm{Et}}\right)$ Real Power flow $\left(\mathrm{P}_{2}\right)$ in case of sudden system disturbance

Table 3

Rating Score for each type of controller during disturbance for the real power $\mathrm{P}_{2}$

\begin{tabular}{|l|l|l|l|}
\hline & $\begin{array}{l}\text { Rise Time } \\
(\text { Sec. })\end{array}$ & $\begin{array}{l}\text { Settling } \\
\text { Time (Sec.) } \\
(2 \%)\end{array}$ & $\begin{array}{l}\text { Overshoot } \\
(\%)\end{array}$ \\
\hline MPC & 0.46 & 0.32 & 2.4 \\
\hline NNMPC & 1.11 & 2.26 & 19.2 \\
\hline
\end{tabular}

\section{Conclusion}

The UPFC based Neural Network Model Predictive Control (NNMPC) has been designed to control the system parameters in the transmission line. The robustness, controllability and the effectiveness of the proposed adaptive controller (NNMPC) has been proven. In addition, the dynamic performance of NNMPC has been tested and compared with another type of adaptive controller scheme called Model Productive Controller (MPC). 


\section{REFERENCES}

[1] G. Shahgholian, M. Mahdavian, M. Janghorbani, I. Eshaghpour, E. Ganji, "Analysis and simulation of UPFC in electrical power system for power flow control", 14th International Conference on Electrical Engineering/ Electronics, Computer, Telecommunications and Inform-ation Technology (ECTI-CON), pp. 6265, 2017.

[2] T.P.G.T.A. Priyankara, M.J.L.R. Fernando, I.A.K. Sandeepa, N.M.K.D. Bandara, H.W.D. Hettiarachchi, K.T.M.U. Hemapala, "Design, simulation and implementation of a UPFC (unified power flow controller) for transmission line model", International Conference on Innovations in Power and Advanced Computing Technologies, pp. 1-6, 2017

[3] H.B. Hatnapure, V.K. Chandrakar, "Power quality improvement by using UPFC", 2nd International Conference for Convergence in Technology (I2CT), pp. 1014-1017, 2017.

[4] Ali Ghasemi and Hossein Shayeghi, "Coordinated design of PSS and TCSC based on Fuzzy controller using global signals", Journal of Power Technologies 98 (1) (2018) 106-120.

[5] R. Hill, G. Wyse and M. Anderson, Animal physiology, $1^{\text {st }}$ ed., Sunderland, MA: Sinauer Associates, Inc, 2004.

[6] L. Gyugyi, "Unified power flow control concept for flexible AC transmission systems ", IEE proceedings $c$ (generation, transmission and distribution), vol. 3, pp. 323-331, 1992.

[7] A. Nabavi-Niaki and M. R. Iravani, "Steady-state and dynamic models of unified power flow controller (UPFC) for power system studies", Presented at 1996 IEEE iPES Winter Meeting, Baltimore, pp. 447-454, 1996.

[8] M. Abido, "Analysis and assessment of statcombased damping stabilizers for power system stability enhancement", Electric Power Systems Research.; vol. 73, ch.2, pp. 177-185, 2005.

[9] M. Abido, A. Al-Awami and Y. Abdel-Magid, "Analysis and design of upfc damping stabilizers for power system stability enhancement", IEEE International Symposium on Industrial Electronics, vol. 3, pp. 2040-2045, 2006.

[10] S. A. Alqallaf, Smart controller design for unified power flow controller using evolutionary optimization algorithms, Kingdom of Bahrain: University of Bahrain. 2005.

[11] B. M.Akesson, H. T. Toivonen, "A Neural network model predictive controller". Journal of Process Control 16, pp. 937-946, 2006.

[12] A. Vasickaninova and M. Bakosova, "Neural Network Predictive Control of a Chemical
Reactor", Proceedings of the 17th International Conference on Process Control, vol. 2, ch. 2, pp. 21-36, 2009.

[13] J. Qin, and T. A. Badgwell, A survey of industrial model predictive control technology, Control Engineering Practice, vol. 11, no. 7, pp. 733-764, 2003.

[14] Wang, L., Model predictive control system

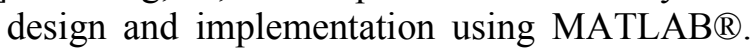
2009: Springer Science \& Business Media.

[15] M.H. Beale, M. T. Hagan, H.B. Demuth, Neural network toolbox ${ }^{\mathrm{TM}}$ user's guide, MA: The MathWorks, Inc.,2014.

[16] M. Luvisotto, Z. Pang and D. Dzung, "Ultra high-performance wireless control for critical applications: Challenges and directions", IEEE Trans. Ind. Informat., vol. 13, no. 3, pp. 14481459, Jun. 2017.

[17] Badwe, A. S., R. D. Gudi, R. S. Patwardhan, S. L. Shah, and S. C. Patwardhan, Detection of Model-Plant Mismatch in MPC Applications, $J$. Process Control, 19, 1305 (2009).

[18] Qin, S. J., and T. A. Badgwell, A Survey of Industrial Model Predictive Control Technology, Control Eng. Practice, 11, 733 (2003).

[19] A. Karimipour and M. Emami, "System Identification and Model Predictive Control of the Chatter Phenomenon in Turning Process", Advances in Science and Technology Research Journal, Volume 13, Issue 3, September 2019, pages 217-228.

[20] G. L. Ji, K. K. Zhang, and Y. C. Zhu, "A method of MPC model error detection", Journal of Process Control, vol. 22, pp. 635-642, 2012.

\section{Creative Commons Attribution License 4.0 (Attribution 4.0 International, CC BY 4.0)}

This article is published under the terms of the Creative Commons Attribution License 4.0 https://creativecommons.org/licenses/by/4.0/deed.en US 